\title{
M31N 2005-09c: a fast Fe II nova in the disk of M 31
}

\author{
D. Hatzidimitriou ${ }^{1}$, P. Reig ${ }^{2}$, A. Manousakis ${ }^{1}$, W. Pietsch ${ }^{3}$, V. Burwitz ${ }^{3}$, and I. Papamastorakis ${ }^{1}$ \\ 1 University of Crete, Department of Physics, PO Box 2208, 71003 Heraklion, Greece \\ e-mail: dh@physics.uoc.gr \\ 2 IESL, Foundation for Research and Technology, 71110 Heraklion, Greece \\ Max-Planck-Institut für extraterrestrische Physik, 85741 Garching, Germany
}

Received 27 June 2006 / Accepted 5 January 2007

\begin{abstract}
Context. Classical novae are quite frequent in M 31. However, very few spectra of M 31 novae have been studied to date, especially during the early decline phase.

Aims. Our aim is to study the photometric and spectral evolution of a M 31 nova event close to outburst.

Methods. Here, we present photometric and spectroscopic observations of M31N 2005-09c, a classical nova in the disk of M 31, using the $1.3 \mathrm{~m}$ telescope of the Skinakas Observatory in Crete (Greece), starting on the 28th September, i.e. about 5 days after outburst, and ending on the 5th October 2005, i.e. about 12 days after outburst. We also have supplementary photometric observations from the La Sagra Observatory in Northern Andalucía, Spain, on September 29 and 30, October 3, 6 and 9 and November 1, 2005. The wavelength range covered by the spectra is from $3565 \AA$ to $8365 \AA$. The spectra are of high $\mathrm{S} / \mathrm{N}$ allowing the study of the evolution of the equivalent widths of the Balmer lines, as well as the identification of non-Balmer lines.

Results. The nova displays a typical early decline spectrum that is characterized by many weak Fe II multiplet emissions. It is classified as a $\mathrm{P}_{\mathrm{fe}}$ nova. From the nova light curve, we have also derived its speed class, $t_{2}=14 \pm 2.5$ days. As the nova evolved the Balmer lines became stronger and narrower. The early decline of the expansion velocity of the nova follows a power law in time with an exponent of $\simeq-0.2$.
\end{abstract}

Key words. galaxies: individual: M 31 - stars: novae, cataclysmic variables

\section{Introduction}

Classical novae are a sub-class of cataclysmic variables, that exhibit outburst magnitudes of 10-20 mag, reaching peak luminosities as high as $M_{V}=-9.5$ for the fastest ones (Warner 1989). Nova outbursts are due to thermonuclear runaways of the hydrogen-rich material that has been accreted onto the white dwarf primary.

The properties of novae may vary between different galaxies (see e.g. Shafter \& Irby 2001). This is an important issue that needs to be clarified before novae can be considered as reliable distance indicators (e.g. Della Valle \& Livio 1995). Moreover, the systematic study of novae in different galaxies in the Local Group is important for improving our understanding of the eruption process. M 31 is an excellent target in this context, due to its proximity and relatively high nova rate.

Novae have been observed in M 31 ever since the pioneering work of Hubble (1929). Several surveys have been published since (cf. Darnley et al. 2004, 2005; Pietsch et al. 2005, and references therein), leading to the discovery of over 470 novae in this galaxy. According to the estimates of Darnley et al. (2005), from the POINT-AGAPE survey, the global nova rate in M 31 is $65_{-15}^{+16} \mathrm{yr}^{-1}$.

Despite the large number of novae discovered in M 31 and their potential importance in achieving a deeper understanding of nova outburst processes in external galaxies, very few spectra of M 31 novae have been published to date. Tomaney \& Shafter (1992) provided the only extensive spectroscopic study of M 31 novae to date, for 9 objects lying in the M 31 Bulge. They further analyzed one of these objects, a remarkably slow nova (Tomaney \& Shafter 1993), which had also been studied spectroscopically earlier (Cowley \& Starrfield 1987).

On September 23, 2005, Quimby et al. (2005) reported the discovery of an optical transient in the direction of M 31, at RA $=00^{\mathrm{h}} 38^{\mathrm{m}} 54.63^{\mathrm{s}}$, Dec $=+40^{\circ} 27^{\prime} 4.2(\mathrm{~J} 2000)$. The location of the transient is shown in Fig. 1, and it appears to be related to the disk of M 31. The detection was based on unfiltered CCD images taken on September 22.17 UT and September 23.21 UT, with the $0.45 \mathrm{~m}$ ROTSE-IIIb telescope at the McDonald Observatory. The magnitude of the transient was about $16.3 \mathrm{mag}$ on the first observation and $16.0 \mathrm{mag}$ on the second. The object was not detected in ROTSE-IIIb data from September 20.18 UT (limiting mag of about 17.6). The transient was spectroscopically confirmed to be a classical nova (Reig et al. 2005). Following the Central Bureau for Astronomical Telegrams (CBAT) nomenclature (http://www.cfa.harvard.edu/iau/CBAT_M31.html), we shall refer to this nova as M31N 2005-09c.

In this paper we have carried out optical observations of M31 2005-09c, in order to monitor its photometric and spectral variability. The spectroscopic observations started on September 28, 2005, i.e., 5 days after outburst, and ended on October 5, 2005, i.e., 12 days after outburst, while the photometric follow up of the nova ended on November 1, 2005.

In Sect. 2, we describe the optical observations, in Sect. 3 the data reduction, in Sect. 4 we present and analyze the results. Finally, in Sect. 5 we summarize our conclusions. 

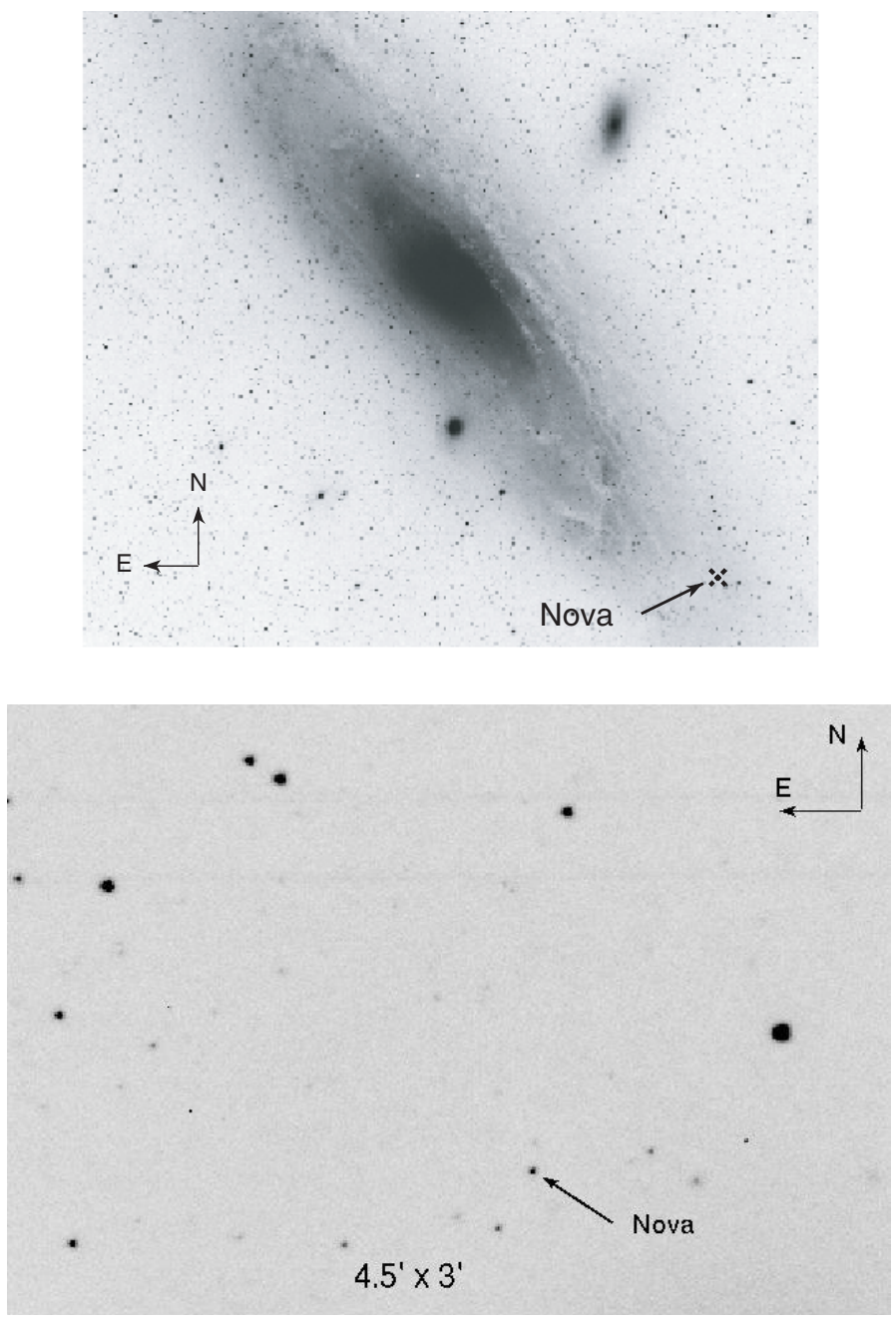

Fig. 1. Finding chart of nova M31N 2005-09c. Its location at RA = $00^{\mathrm{h}} 38^{\mathrm{m}} 54.63^{\mathrm{s}}$, Dec $=+40^{\circ} 27^{\prime} 34.2^{\prime \prime}(\mathrm{J} 2000)$, is marked on the M $31 \mathrm{im}-$ age taken from NASA's Sky View Virtual Observatory, based on photographic data obtained using the Oschin Schmidt Telescope on Palomar Mountain.

\section{Observations}

The spectroscopic observations used in this study were carried out during four nights, on September 28 and 29, 2005 and on October 3 and 5, 2005, using the 1.3-m Ritchey-Cretien telescope at Skinakas Observatory, located on the island of Crete (Greece).

The telescope was equipped with a $2000 \times 800$ ISA SITe CCD camera and a 1302 lines/mm grating, blazed at $5500 \AA$. Spectra were obtained in four overlapping wavelength regions in order to include as many spectral lines of interest as possible. The overall spectral range covered was from $3565 \AA$ to $8365 \AA$ A. The nominal dispersion was $1.04 \AA$ Apixel. Exposure times ranged from 600 to $3600 \mathrm{~s}$, depending on brightness of the object and on weather and seeing conditions. Each on-target exposure was followed by an arc calibration exposure. The log of the observations is given in Table 1.

The acquisition data which were unfiltered, were used to derive approximate magnitudes of the nova, as will be described in detail in the next section.

We also have some complimentary photometric observations from the La Sagra Observatory (run by Observatorio Astronomic de Mallorca, hereafter, OAM) in north-east Andalucía, Spain.
Table 1. Log of spectroscopic observations of M31N 2005-09c.

\begin{tabular}{cccc}
\hline \hline Date & $\begin{array}{c}\text { JD } \\
2453600.0+\end{array}$ & $\begin{array}{c}\text { Spectral } \\
\text { Range }(\AA)\end{array}$ & $\begin{array}{c}\text { Exposure } \\
\text { time }(\mathrm{s})\end{array}$ \\
\hline 28 Sep. 05 & 42.307 & $4750-6825$ & 600 \\
& 42.315 & $4750-6825$ & 1800 \\
& 42.338 & $4750-6825$ & 3000 \\
29 Sep. 05 & 43.304 & $3565-5675$ & 3000 \\
& 43.417 & $3565-5675$ & 3500 \\
& 43.491 & $3565-5675$ & 3500 \\
& 43.349 & $5090-7160$ & 3000 \\
& 43.542 & $5090-7160$ & 3000 \\
03 Oct. 05 & 43.260 & $4750-6825$ & 3000 \\
& 47.390 & $4750-6825$ & 3300 \\
05 Oct. 05 & 47.446 & $6315-8365$ & 3300 \\
& 49.264 & $3565-5675$ & 3600 \\
& 49.312 & $3565-5675$ & 3600 \\
& 49.370 & $4750-6825$ & 3600 \\
& 49.414 & $4750-6825$ & 3600 \\
& 49.471 & $6315-8365$ & 3600 \\
\hline
\end{tabular}

These observations were obtained on September 29 and 30, October 3, 6 and 9, and November 1, 2005, using a Celestron CGE1400 14 inch telescope with a Hyperstar focal reducer and a 10XME camera with a KODAK KAF-3200 ME CCD chip. The pixel scale was $2.13^{\prime \prime}$ per $6.8 \mu$ pixel, with a total field of view of $77.4^{\prime}$ by $52.1^{\prime}$. The images were white light images (i.e. no filter was used). The dates and times of the observations are listed in Table 2 along with the derived magnitudes of the nova (see next section).

\section{Data reduction}

\subsection{Photometry}

\subsubsection{Skinakas observatory}

The "white light magnitudes" of the nova M31N 2005-09c were converted to $R$ magnitudes by comparing them against the $R$ magnitudes of USNO-B1 (Monet et al. 2003) stars in the acquisition fields. This was achieved by using 9 bright USNO-B1.0 stars, present in the acquisition field, as secondary standards. For each image, a separate calibration curve was produced from the instrumental magnitudes of the 9 stars and the corresponding USNO-B1.0 $R$ magnitudes ${ }^{1}$. The calibration curve was approximated by a linear fit, from which we then derived the $R$ magnitude and associated error of the nova. These estimates are presented in the upper panel of Table 2.

One important caveat regarding the accuracy of the derived R-magnitudes, is that the nova is an emission line object, while the USNO calibration stars are mostly "normal" stars. In order to at least partly address this problem, we compared the colour distribution of known novae (van den Bergh \& Younger 1987, for novae near maximum; and Szkody 1994, for older novae) to the colour distribution of the calibrating stars. The colour range of the calibrating stars covers that of novae (near maximum and older ones), although the peaks of the colour distributions of the calibrating stars and of novae are somewhat different, with the novae being bluer in the mean than the calibrating stars. There is a small colour term in the magnitude residuals of the calibrators, indicating that a correction of 0.09 mag should be applied to

1 The USNO-B1 magnitudes are tied to the Jonhson-Kron-Cousins system (cf. Bucciarelli et al. 2001), therefore the quoted $R$ magnitude is "Cousins R". 
Table 2. Photometry from Skinakas Observatory and from the La Sagra Observatory.

\begin{tabular}{ccc}
\hline \hline Date & JD & $R$ \\
& $2453600.0+$ & $\mathrm{mag}$ \\
\hline \multicolumn{3}{c}{ Skinakas Observatory } \\
28.09 .05 & 42.303 & $17.44 \pm 0.22$ \\
29.09 .05 & 43.251 & $17.49 \pm 0.14$ \\
& 43.407 & $17.51 \pm 0.18$ \\
& 43.481 & $17.48 \pm 0.22$ \\
03.10 .05 & 47.262 & $17.54 \pm 0.17$ \\
& 47.316 & $17.47 \pm 0.12$ \\
05.10 .05 & 49.255 & $17.65 \pm 0.20$ \\
& 49.366 & $17.72 \pm 0.24$ \\
\multicolumn{3}{c}{ La Sagra Observatory } \\
15.09 .05 & 29.856 & $>20.5$ \\
29.09 .05 & 43.190 & $17.18 \pm 0.14$ \\
30.09 .05 & 44.127 & $17.44 \pm 0.14$ \\
03.10 .05 & 47.966 & $17.80 \pm 0.16$ \\
06.10 .05 & 50.184 & $17.73 \pm 0.18$ \\
09.10 .05 & 53.012 & $18.47 \pm 0.22$ \\
01.11 .05 & 76.949 & $>20.2$ \\
\hline
\end{tabular}

bluer objects. This correction falls well within the magnitude error margins quoted in Table 1 . Thus, the size of the $R$-magnitude error that we derived indeed should include the error due to the unknown spectral type of the specific nova. In any case, it must be emphasized that all of the nova data used to provide the lightcurve discussed in Sect. 4.1, including the La Sagra (see below) and the Quimby et al. (2005) data are calibrated against USNO-R magnitudes. Therefore, even if there is a zero-point error in the lightcurve, the speed class derived from the lightcurve should be valid.

\subsubsection{La Sagra observatory, OAM}

For each night, a median image was created from 15 individual images of $60 \mathrm{~s}$ exposure each. These median images were then reduced and analyzed using the Astrometrica program (by Herbert Raab, www.astrometrica.at), which automatically selects stars and compares them to the USNO-B1.0 catalogue, in order to provide calibration curves for the photometry. The resulting $R$ magnitudes and associated errors for the nova M31N 2005-09c are listed in the lower panel of Table 2.

For the nights of September 29, 2005 and October 3, 2005, there are magnitude estimates for the nova from both observatories. In the former, the difference between the magnitudes $\left(R_{\text {Skinakas }}-R_{\text {OAM }}\right)$ obtained closest in time is $0.30 \pm 0.22 \mathrm{mag}$, while in the latter, $-0.33 \pm 0.20 \mathrm{mag}$. Thus, there is no evidence of a systematic difference between the photometry from the two telescopes, and the random errors are within the expected range.

As can be seen in Table 2, for the nights of September 15, 2005 and November 1, 2005, only lower limits for the magnitude of the nova could be derived. Actually, all available OAM images of the field were examined, both before the outburst, going back to September 25, 2005 and after the last date shown in Table 2 (November 1, 2005), until the December 1, 2005. The object was not detected on any of these images (i.e. it was fainter than $R \simeq 20.5 \mathrm{mag}$ ).

\subsection{Spectroscopy}

The reduction of the spectra was performed using the STARLINK Figaro package (Shortridge et al. 2001). The frames were bias

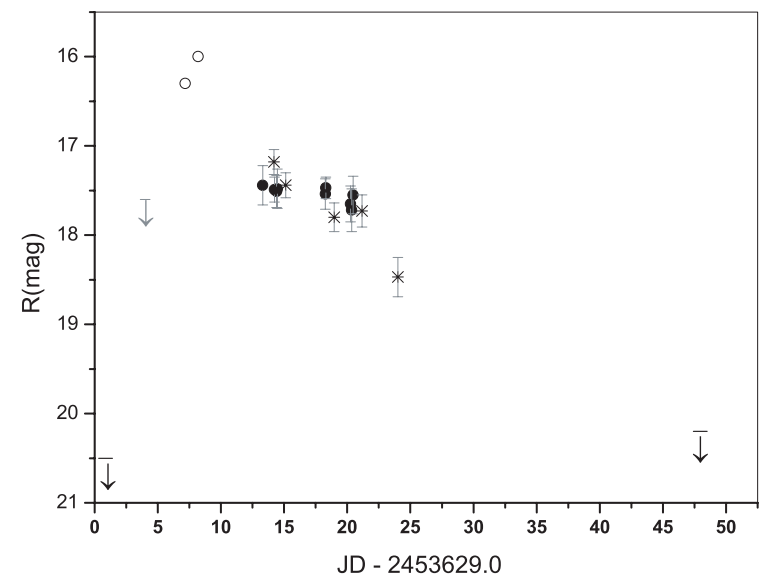

Fig. 2. The light curve of the nova between September 15, 2005 and November 1, 2005. Filled circles correspond to the Skinakas measurements and asterisks to the La Sagra measurements, summarized in Table 2. The down-arrows show lower limits of the nova's magnitude. Open circles correspond to approximate magnitudes given by Quimby et al. (2005).

subtracted, flat fielded and corrected for cosmic ray events. The 2D spectra were subsequently sky subtracted using the POLYSKY command. A spatial profile was then determined for each 2D spectrum, and, finally, the object spectra were optimally extracted using the algorithm of Horne (1986), with the OPTEXTRACT routine. Arc spectra were then extracted from the arc exposures, using exactly the same profiles as for the corresponding object spectra. The arc spectra were subsequently used to calibrate the object spectra.

The full width at half maximum (FWHM) of the arc lines were also used to estimate the instrumental broadening (resolution) of the spectral lines. The instrumental broadening depends on the position of the line on the CCD chip. When the line is near the edge the instrumental width is wider. Typical values are $6 \AA$ at the edges and about half that value in the center of the chip. The quoted values of the FWHM of the spectral lines reported here were corrected for this effect, taking into account their position on the chip.

\section{Results}

\subsection{Lightcurve}

In Fig. 2, we present an estimate of the light curve of the nova, based on the magnitudes of Table 2 and on the magnitude estimates of Quimby et al. (2005), mentioned in Sect. 1. The former are presented as filled circles (from Skinakas Observatory) and asterisks (La Sagra Observatory, OAM), along with their associated errors, as given in Table 2. The Quimby et al. values are shown as open circles. The ROTSE-IIIb data on which these values were based were unfiltered (roughly 400-800 nm). The magnitudes reported in Quimby et al. (2005) were calibrated against the USNO-A1.0 $R$ band (Quimby 2006, private communication). The arrows indicate the lower detection limits of the 20th of September observation of Quimby et al. (limiting magnitude 17.6, grey arrow), and of the lower detection limits mentioned in Table 2, from OAM (black arrows).

It appears that the nova had a magnitude around $R \simeq$ 16.0 mag near maximum light, which corresponds to an absolute magnitude of $M_{R} \simeq-8.6$, using $(m-M)_{V}=24.47 \pm 0.07$ 


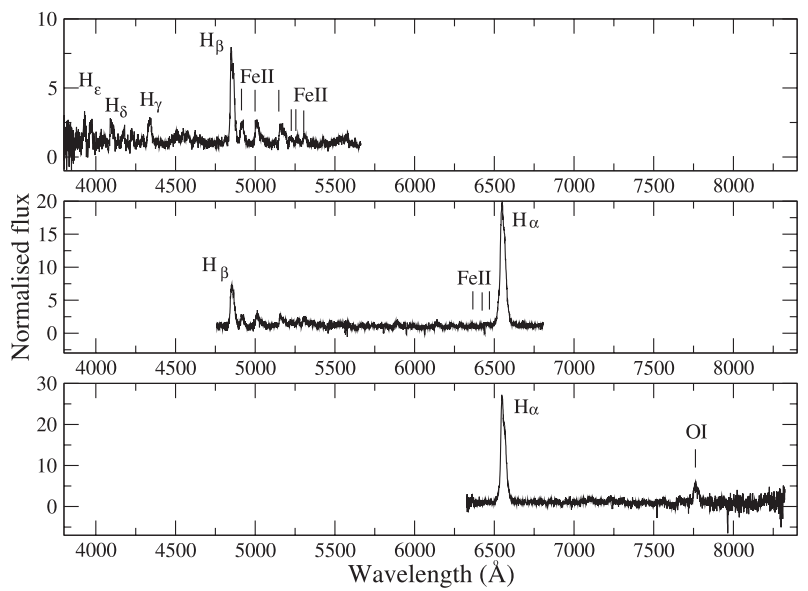

Fig. 3. The optical spectrum of the nova obtained in three different wavelength regions, covering 3700-8200 A. Top and middle panels correspond to the average of three and two observations respectively, obtained on September 29, 2005, while the bottom panel corresponds to one near-IR observation obtained on October 3, 2005. All spectra have been normalized to unity.

and $E(B-V)=0.06$ from Holland (1998) and the conversion $A_{R} / A_{V}=0.748$ from Rieke \& Lebofsky (1985).

The "speed class" of a classical nova is often used to describe the overall timescale of an eruption and to classify a nova. The speed class, $t_{2}$, is the number of days that a nova takes to fade by two magnitudes below maximum light (e.g. Darnley et al. 2005). According to the light curve of Fig. 2, the nova M31N 2005-09c has faded by about two magnitudes in $t_{2}=14 \pm 2.5$ days (it was about 16th mag, on the 23rd September and about 18 mag between the 6th and 9th October). The error is estimated on the basis of the quoted errors in $R$ and the frequency of observations.

Using the recent maximum magnitude versus rate of decline (MMRD) relationship of Downes \& Duerbeck (2000), $M_{V}=(-11.32 \pm 0.44)+(2.55 \pm 0.32) \log \left(t_{2}\right)$, we estimate that for the derived rate of decline of $t_{2}=14 \pm 2.5$ days, $M_{V}$ at maximum would be expected to be around $\simeq-8.4 \pm 0.6$. Our estimate of $M_{R}$ is $\simeq-8.6$, i.e. very close to the value derived from the MMRD relationship for $M_{V}$. Note, that given that novae close to maximum are blue $\left((B-V)_{0} \simeq 0.23\right.$; van den Bergh \& Younger 1987; Downes \& Duerbeck 2000), $(V-R)_{0}$ is expected to be small, well within the quoted errors.

\subsection{Line identification}

Figure 3 shows examples of spectra obtained for the transient, in three different wavelength regions. The top panel shows the spectrum from 3700-5675 $\AA$ (average of three spectra obtained on September 29), the middle panel from 4750-6825 $\AA$ (average of two spectra obtained on September 29, 2005) and the bottom panel from 6315-8365 $\AA$ (one observation obtained on October 3, 2005). All spectra have been normalized to unity.

The lines of the Balmer series up to $\mathrm{H} \epsilon$ appear strongly in emission. The spectra are also characterized by Fe II emission, forbidden and permitted. The lines identified are listed in Table 3. Column 1 gives the line identification, with the multiplet number in brackets and forbidden transitions indicated by square brackets. Column 2 gives the theoretical rest wavelength of the line (from Thackeray 1952) and Col. 3 the observed wavelength of the line.
Table 3. Identified emission lines.

\begin{tabular}{rcc}
\hline \hline $\mathrm{ID}^{1}$ & $\lambda_{\mathrm{o}}(\AA)$ & $\lambda_{\mathrm{obs}}(\AA)$ \\
\hline $\mathrm{Fe} \mathrm{II}(3)$ & 3930.3 & 3931.3 \\
$\mathrm{H} \epsilon$ & 3970.1 & 3970.0 \\
$\mathrm{H} \delta$ & 4101.7 & 4101.0 \\
$\mathrm{H} \gamma$ & 4340.5 & 4341.2 \\
{$[\mathrm{Fe} \mathrm{II}](20)$} & 4852.7 & 4853.2 \\
$\mathrm{H} \beta$ & 4861.3 & 4861.4 \\
$\mathrm{Fe} \mathrm{II}(42)$ & 4923.9 & 4922.9 \\
$\mathrm{Fe} \mathrm{II}(42)$ & 5018.4 & 5018.2 \\
$\mathrm{Fe} \mathrm{II}(42)$ & 5169.0 & 5168.3 \\
$\mathrm{Fe} \mathrm{II}(49)$ & 5234.6 & 5235.2 \\
{$[\mathrm{Fe} \mathrm{II}](18)$} & 5268.9 & 5269.8 \\
{$[\mathrm{Fe} \mathrm{II}](18)$} & 5273.4 & 5276.4 \\
$\mathrm{Fe} \mathrm{II}(41)$ & 5284.1 & 5283.1 \\
$\mathrm{Fe} \mathrm{II}(49)$ & 5327.1 & 5326.5 \\
$\mathrm{Fe} \mathrm{II}$ & 5894.1 & 5894.6 \\
$\mathrm{Fe} \mathrm{II}(74)$ & 6147.7 & 6150.0 \\
$\mathrm{Fe} \mathrm{II}(74)$ & 6238.4 & 6241.6 \\
$\mathrm{H} \alpha$ & 6562.8 & 6560.4 \\
$\mathrm{O} \mathrm{I}$ & 7773.0 & 7774.8 \\
\hline
\end{tabular}

${ }^{1}$ Suggested identification is followed by the multiplet number in brackets. Forbidden lines are distinguished by square brackets.

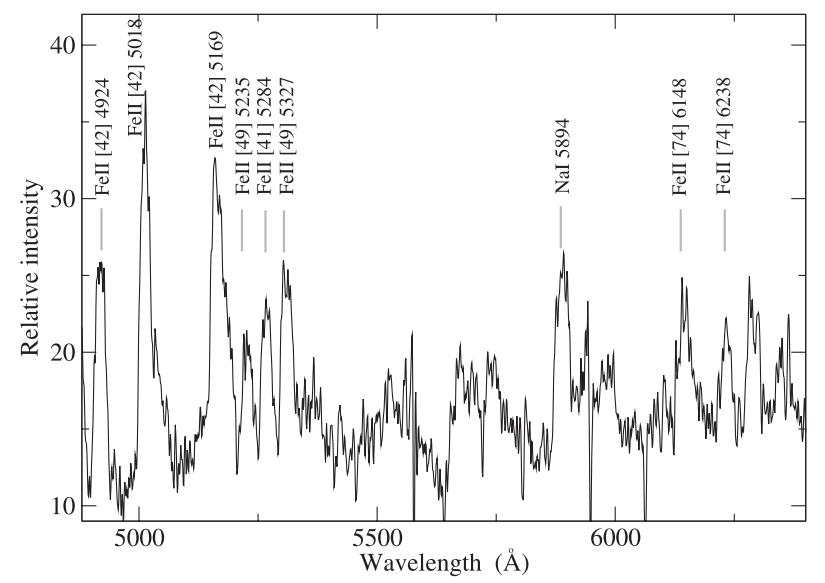

Fig. 4. Identification of Fe II lines between 4800-6400 A.

Most of the Fe II lines lie between the $\mathrm{H} \alpha$ and $\mathrm{H} \beta$ lines. Figure 4 shows the spectrum of the nova in this region, i.e. between 4800 and $6400 \AA$, with the identified lines clearly marked.

Apart from the Balmer and Fe II lines, two more lines were also seen in emission, namely, Na ID $\lambda 5894 \AA$ (shown in Fig. 4) and O I $\lambda 7773 \AA$ (shown in Fig. 2).

In conclusion, the nova displays a typical early decline spectrum that is characterized by many weak Fe II multiplet emissions. Following the Tololo Nova Spectral Classification System (Williams et al. 1991; Williams et al. 1994), we classify the nova as a $\mathrm{P}_{\mathrm{fe}}$ nova. The nova is undoubtedly in the permitted lined phase $(\mathrm{P})$, as the strongest non-Balmer emission is a permitted transition. The subclass is assigned from the strongest non-Balmer line in the spectrum, which is clearly Fe II $\lambda 5018 \AA$, $\lambda 5169 \AA$. The presence of the O I line at $7773 \AA$, suggests that the nova is close to the OI flash, which is expected at about 2.5 mag below maximum light (e.g. Tomaney \& Shafter 1992). On the spectrum of October 5, 2005, there is clear indication of the O I $\lambda 6300 \AA$ line. This line is expected (at least for galactic classical novae) to "turn on" at 2.6 mean magnitudes from maximum (Tomaney \& Shafter 1992). 

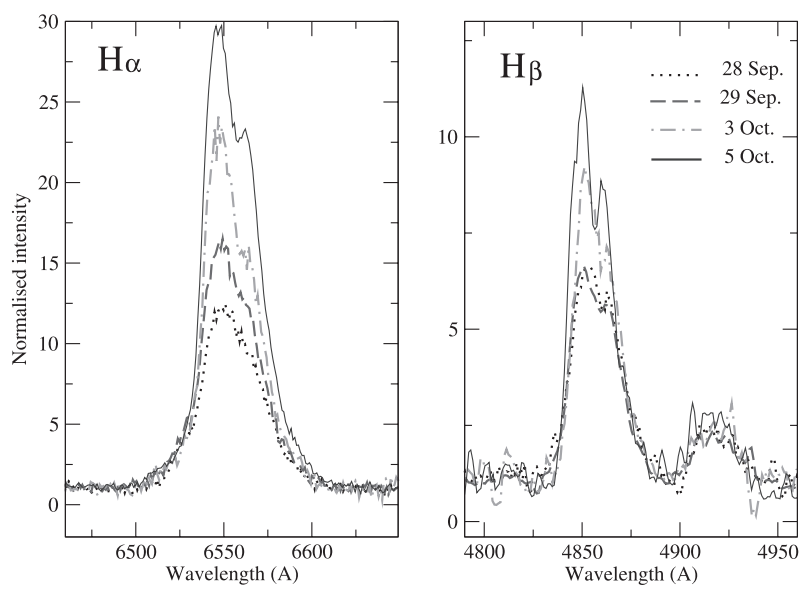

Fig. 5. The evolution of the intensity and profile of the $\mathrm{H} \alpha$ and $\mathrm{H} \beta$ lines. The spectra were normalized to the neighboring continuum.

Table 4. Equivalent widths of $\mathrm{H} \alpha$ and $\mathrm{H} \beta$ emission lines.

\begin{tabular}{cccccc}
\hline \hline Date & \multirow{2}{*}{ MJD } & \multicolumn{2}{c}{$E W(\AA)$} & \multicolumn{2}{c}{$F W H M^{1}\left(\mathrm{~km} \mathrm{~s}^{-1}\right)$} \\
& & $\mathrm{H} \alpha$ & $\mathrm{H} \beta$ & $\mathrm{H} \alpha$ & $\mathrm{H} \beta$ \\
\hline 28 Sep. 05 & 53642 & $550 \pm 40$ & $160 \pm 5$ & $1800 \pm 20$ & $1760 \pm 80$ \\
29 Sep. 05 & 53643 & $630 \pm 40$ & $160 \pm 5$ & $1735 \pm 20$ & $1700 \pm 70$ \\
03 Oct. 05 & 53647 & $900 \pm 100$ & $185 \pm 8$ & $1590 \pm 30$ & $1500 \pm 60$ \\
05 Oct. 05 & 53649 & $1100 \pm 100$ & $210 \pm 10$ & $1610 \pm 30$ & $1490 \pm 60$ \\
\hline
\end{tabular}

${ }^{1}$ The quoted values have been corrected for instrumental broadening.

\section{3. $H \alpha$ and $H \beta$ equivalent width evolution}

Shortly after eruption, novae develop strong and broad $\left(\geq 1000 \mathrm{~km} \mathrm{~s}^{-1} \mathrm{H} \alpha\right.$ emission lines that fade slowly (Shafter \& Irby 2001). Figure 5 displays the $\mathrm{H} \alpha$ and $\mathrm{H} \beta$ emission lines of the M 31 nova, and their evolution during the period of the observations. Both lines show rather asymmetric saddle shaped profiles, with the blue component more prominent than the red. As the nova evolves the line width decreases while their intensity increases and the double peak profile becomes more distinct. Very similar behavior of the $\mathrm{H} \alpha$ and $\mathrm{H} \beta$ emission lines has been observed during the early decline phase of Nova V382 Vel 1999 (Della Valle et al. 2002).

Table 4 gives the equivalent width (EW) and full width at half maximum (FWHM) of the $\mathrm{H} \alpha$ and $\mathrm{H} \beta$ lines. The main source of error in the calculation of the equivalent width is the determination of the continuum. Thus the procedure was repeated five times, selecting different continuum points in each case. The average values of the derived equivalent width and the associated standard deviations are reported in Table 4 . They range from $550 \AA$ to $1100 \AA$ for $\mathrm{H} \alpha$ and from $160 \AA$ to $210 \AA$ for $\mathrm{H} \beta$.

The FWHMs were corrected for instrumental broadening according to the position of the line in the CCD chip by using a near-by arc line. The evolution of the FWHM during the period of our observations is shown in Fig. 6. This early decline of the expansion velocity of the nova follows a power law in time with an exponent of $-0.19 \pm 0.04$ for the $\mathrm{H} \alpha$ line, and of $-0.28 \pm 0.03$ for the $\mathrm{H} \beta$ line, which are comparable to the values found for the early decline of Nova V382 Vel 1999 (Della Valle et al. 2002).

\section{Summary}

We have obtained photometric and spectroscopic observations of M31N 2005-09c, a classical nova in the disk of M 31, using the

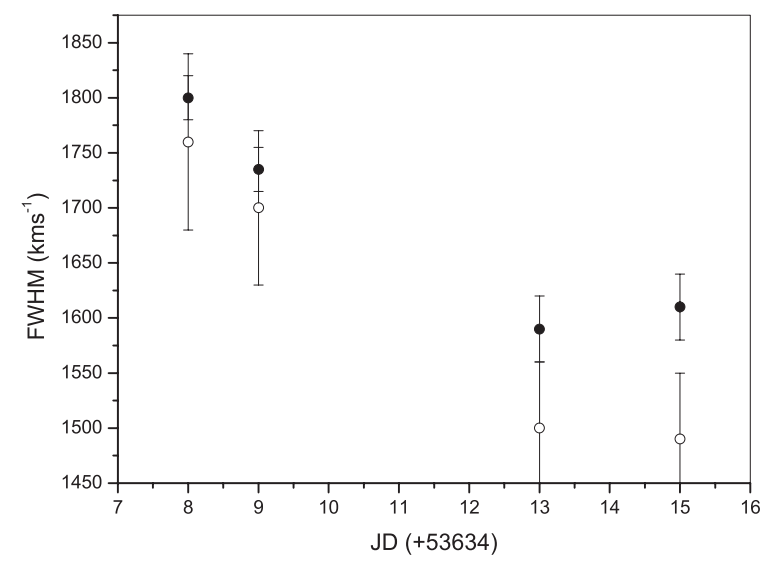

Fig. 6. The evolution of the FWHM of the $\mathrm{H} \alpha$ (filled circles) and $\mathrm{H} \beta$ lines (open circles). JD reference is 53634.

$1.3 \mathrm{~m}$ telescope of the Skinakas Observatory in Crete (Greece), starting on September 28, 2005, i.e. about 5 days after outburst, and ending on the October 5, 2005, i.e. about 12 days after outburst. We also used supplementary photometric observations from the La Sagra Observatory in Northern Andalucía, Spain, on September 29 and 30, October 3, 6 and 9 and November 1, 2005. The nova displays a typical early decline spectrum that is characterized by many weak Fe II multiplet emissions. It is classified as a $\mathrm{P}_{\mathrm{fe}}$ nova. From the nova light curve, we have also derived its speed class, $t_{2}=14 \pm 2.5$ days. The full width at half maximum of the $\mathrm{H} \alpha$ and $\mathrm{H} \beta$ lines is $\simeq 1700 \mathrm{~km} \mathrm{~s}^{-1}$ and $\simeq 1600 \mathrm{~km} \mathrm{~s}^{-1}$, respectively. The early decline of the expansion velocity of the nova follows a power law in time with an exponent of $\simeq-0.2$.

Acknowledgements. We thank the referee, Dr. A. Evans, whose comments and suggestions improved the original version of this paper. The authors are thankful to T. Koutentakis who helped with the observations at Skinakas Observatory and Nicolás Morales for observations at the (OAM) La Sagra observatory. Sincere thanks are also due to Dr. R. Quimby for providing us with additional information regarding the nova photometry given in Quimby et al. (2005).

\section{References}

Bucciarelli, B., Garcia Yus, J., Casalegno, R., et al. 2001, A\&A, 368, 335 Cowley, A. P., \& Starrfield, S. 1987, PASP, 99, 854

Darnley, M. J., Bode, M. F., Kerins, E., et al. 2004, MNRAS, 353, 571 Darnley, M. J., Bode, M. F., Kerins, E., et al. 2006, MNRAS, 369, 257 Della Valle M., \& Livio, M. 1995, ApJ, 452, 704

Della Valle, M., Pasquini, L., Daou, D., \& Williams, R. E. 2002, A\&A, 390, 155 Downes, R. A., \& Duerbeck, H. W. 2000, AJ, 120, 2007

Holland, S. 1998, AJ, 115, 1916

Horne, K. 1986, PASP, 98, 609

Hubble, E. 1929, ApJ, 69, 103

Monet, D. G., Levine, S. E., Canzian, B., et al. 2003, AJ, 125, 984

Pietsch, W., Fliri, J., Freyberg, M. J., et al. 2005, A\&A, 442, 879

Quimby, R., Mondol, P., Hoeflich, P., et al. 2005, ATel, 611

Reig, P., Hatzidimitriou, D., Manousakis, A., et al. 2005, ATel, 618

Rieke, G. H., \& Lebofsky, M. J. 1985, ApJ, 288, 618, 1

Shafter, A. W., \& Irby, B. K. 2001, ApJ, 563, 749

Shortridge, K. 2001, Software in Astronomy, in The Encyclopedia of Astronomy and Astrophysics, ed. P. Murdin (London: Institute of Physics Publishing) Szkody, P. 1994, AJ, 108, 639

Thackeray, A. D. 1952, MNRAS, 113, 212

Tomaney, A. B., \& Shafter, A. W. 1992, ApJS, 81, 683

Tomaney, A. B., \& Shafter, A. W. 1993, ApJ, 411, 640

van den Bergh, S., \& Younger, P. F. 1987, A\&AS, 70, 125

Warner, B. 1989, in Classical novae, ed. M. F. Bode \& A. Evans (Chichester: Wiley), 1

Williams, R. E., Hamuy, M., Phillips, M. M., et al. 1991, ApJ, 376, 721

Williams, R. E., Phillips, M. M., \& Hamuy, M. 1994, ApJS, 90, 297 